\title{
Acerca de la distinción entre la capacidad de juzgar determinante y reflexionante en Kant*
}

\section{Carlos Mendiola}

$\mathrm{E}$

1 propósito de este trabajo consiste en exponer la distinción entre capacidad de juzgar determinante y capacidad de juzgar reflexionante ${ }^{1}$ que Immanuel Kant presenta en la Crítica de la capacidad de juzgar (1790).

Esta exposición lo que persigue es mostrar el punto distintivo entre las dos capacidades. Además pretende que la capacidad de juzgar reflexionante ocupa un lugar central en la obra crítica kantiana, porque ya desde la Crítica de la razón pura (1781/1787) aparece la necesidad de proponer la capacidad de juzgar reflexionante. ${ }^{2}$

Para desarrollar la exposición de esta distinción comenzaremos por mostrar su formulación nominal en la Crítica de la capacidad de juzgar, dado que, como veremos, no podemos comprender la distinción en su sentido literal. Más tarde trataremos de abrir la posibilidad de comprender la capacidad

* Agradezco a E. Lazos y a C. Santos, por sus comentarios.

${ }^{1}$ Traduzco die reflektierende Urteilskraft por la capacidad de juzgar reflexionante y die bestimmende Urteilskraft por la capacidad de juzgar determinante, aunque comúnmente se traducen como "Juicio reflexionante" y "Juicio determinante", marcando su distinción respecto del juicio (Urteil) por medio de la jota mayúscula. La razón de esta traducción radica en que mi propósito es mostrar que la distinción que hace Kant depende de la relación que guardan las capacidades con los juicios. Esto es, que el juicio determinante necesita de la participación de las dos capacidades y el juicio reflexionante sólo de la capacidad de juzgar reflexionante. Todas las citas de las obras de Kant son comparadas, en cuanto a su traducción castellana, con la edición de las Werkausgabe. Herausgegeben von Wilhelm Weischedel. Las referencias a esta edición aparecerán entre corchetes, con paginación y volumen.

${ }^{2}$ El argumento que aparece aquí es sólo una parte de un trabajo más extenso, cuya primera parte muestra la ubicación de esta distinción en la obra crítica: 1) la importancia de una exposición de la formulación de la distinción; 2) ubicación de la distinción en la obra crítica; 3) ubicación de la distinción en la Crítica de la capacidad de jugar, y 4) ubicación de la distinción en el sistema. 
de juzgar reflexionante; con esta intención dividiremos la exposición en dos grandes secciones. En la primera, buscaremos dirigirla hacia la capacidad de juzgar reflexionante, mostrando lo que Kant dice acerca de la capacidad de juzgar determinante y lo que calla acerca de la otra en las obras anteriores a la tercera Crítica. Con tal motivo esta primera sección expondrá lo dicho en la Crítica de la razón pura sobre la capacidad de juzgar determinante y su distinción respecto del juicio de percepción (como el antecedente más cercano al juicio reflexionante), ${ }^{3}$ según la manera en que aparece formulado en los Prolegómenos a toda metafísica que haya de poder presentarse como ciencia (1783), como juicio de experiencia y juicio de percepción. La segunda sección se encargará de exponer la capacidad de juzgar reflexionante mostrando sus elementos distintivos en relación con la otra capacidad.

\section{Formulación nominal de la distinción en la Crítica de la capacidad de juzgar}

La formulación nominal dice que puede distinguirse la "capacidad de juzgar" en dos modos de su ejercicio y, por consiguiente, en dos juicios que son productos de aquellos dos modos. El primer modo de ejercicio es la capacidad determinante con su producto, el juicio determinante, y el segundo modo de ejercicio es la capacidad reflexionante con su producto, el juicio reflexionante.

La capacidad determinante - de acuerdo con esta formulación- subsume un particular bajo un universal ya dado. Esta subsunción consiste en conformar al particular por medio de una categoría. ${ }^{4} \mathrm{El}$ particular no posee objetividad hasta que no adquiere esta conformidad. La conformidad no es más que

${ }^{3} \mathrm{Cf}$. Immanuel Kant, Prolegómenos a toda metafísica que haya de poder presentarse como ciencia. Trad. de Mario Caimi. Madrid, Istmo, 1999, pp. 124-127 (edición bilingüe). También puede confrontarse esta lectura con: Laura Mues de Schrenk, Interpretación del concepto experiencia en los Prolegómenos. México, UNAM, 1983, pp. 66-73; Rudolf A. Makkreel, Imagination and Interpretation in Kant. The Hermeneutical Import of the Critique of Judgement. Chicago, Universidad de Chicago, 1990, pp. 33-34, y Fernando Montero, Mente y sentido interno en la Crítica de la razón pura. Barcelona, Crítica, 1989, p. 143.

4 "Las categorías son las formas fundamentales de la síntesis de los datos para la unidad de la experiencia objetiva - condiciones a priori, constituyentes de la experiencia- las cuales valen necesariamente por esto para toda experiencia posible". Traducción mía. (Rudolf Eisler, Kant Lexikon Nachschlagewerk zu Kants sämtlichen Schriften / Briefen und Handschriftlichem Nachlass. Hildscheim, Georg Olms, 1969, p. 282). 
un concepto que nos permite hacer inteligible aquel particular: un juicio ${ }^{5}$ que no es otra cosa que el enlace entre categoría y predicado.

En cambio la capacidad reflexionante debe producir la categoría o el universal para un particular dado. Esta capacidad tiene que encontrar la categoría que permita conformar al particular. Lo único con lo que se cuenta, en este caso, es el particular. Y lo que interesa en ambos modos de ejercicio es llegar al concepto que haga inteligible al particular: el juicio.

La capacidad de juzgar en general es la capacidad de pensar lo particular en cuanto contenido bajo lo universal. Si lo universal (la regla, el principio, la ley) es dado, la capacidad de juzgar, que subsume bajo él lo particular [...] es determinante. Si lo particular es dado, para lo cual debe encontrar ella lo universal, la capacidad de juzgar es sólo reflexionante. ${ }^{6}$

La capacidad determinante ha sido explicada y demostrada en la Crítica de la razón pura. La particularidad es la conformidad que dan las formas de la sensibilidad (espacio y tiempo) en la intuición. Si la intuición es empírica cuenta con una diversidad de sensaciones, que no es más que el producto de la afección que recibe la sensibilidad. Si, en cambio, la intuición es pura, sólo cuenta con la configuración, como una autoafección, de las formas de la sensibilidad, que ofrecen una estructura vacía de sensación. La categoría somete el actuar de la espontaneidad del entendimiento para conformar la particularidad (intuición) al concepto. Intuición y categoría son abstracciones que se generan en el análisis de algo único que llamamos conocimiento. La principal tarea de estas abstracciones consiste en permitirnos decir que no hay en parte alguna mera intuición o mera categoría, sino sólo aquello único (el conocimiento) del que intuición y categoría son elementos sólo separados por abstracción.

${ }^{5}$ Kant define: "juicio es la representación de la unidad de conciencia de diferentes representaciones o la representación de su relación en cuanto forma de un concepto" (I. Kant, Lógica. Introducción al estudio de la filosofía. México, Editorial Nacional, 1981, p. 105 [p. 531, vol. vI]).

${ }^{6}$ I. Kant, Crítica de la facultad de juzgar. Trad. de Pablo Oyarzún. Caracas, Monte Ávila, 1991, p. 90 [p. 87, vol. x]. La primera versión de la introducción, aunque expresa de manera diferente la distinción, contiene esencialmente la misma idea. "La capacidad de juzgar puede ser considerada ya como una capacidad para reflexionar según un cierto principio sobre una representación dada, en pro de un concepto que por ese medio es posible, ya como una capacidad para determinar, por una representación empírica dada, un concepto que hace de fundamento. En el primer caso es ella la capacidad de juzgar reflexionante, en el segundo, la capacidad de juzgar determinante" (Ibid., p. 35 [p. 24, vol. x]). 
La sensación en la intuición empírica ofrece la materia sobre la que se aplica la categoría. Como simple materia no es más que diversidad caótica, que la sensibilidad recibe ordenándola bajo estructuras dadas (espacio y tiempo), pero estas estructuras por sí solas no alcanzan su unificación. Ellas sólo ofrecen en la intuición empírica una diversidad ordenada. Espacio y tiempo, como estructuras que condicionan todo ordenamiento de la materia (dada por la sensación), pueden a su vez ofrecer un ordenamiento de lo que podría presentarse como materia sin tener que contenerla, tan sólo contemplando su posibilidad. Este ordenamiento es la intuición pura, que ofrece la estructura posible de una diversidad dada en las formas de la sensibilidad y no unificada.

En cambio, la categoría sí unifica esa diversidad que ofrece la intuición, ya que la categoría es una unidad vacía de contenido. Unidad (categoría) y diversidad (intuición) constituyen las partes de la síntesis (juicio) del conocimiento. Esta síntesis es producto de la capacidad determinante. En cuanto se le presenta a esta capacidad determinante el primer eslabón en una afección o autoafección (sensación o configuración formal) se desemboca hasta su última consecuencia que es la misma síntesis. La capacidad determinante tiene una manera de proceder automática. Gracias a que cuenta ya con una categoría puede aplicarla a todo particular. ${ }^{7}$

La objetividad del juicio se cifra en su determinación por el espacio y el tiempo, y su verdad por las categorías que determinan la síntesis que ofrece conocimiento. ${ }^{8}$ La categoría debe poderse aplicar a todo posible particular, ya que no puede conocerse de otra manera que por esta aplicación. El particular queda subsumido por la categoría, de tal manera que el sustento y correlato del conocimiento son las leyes de la naturaleza que corresponden a estas categorías, y que determinan su aplicación en el juicio. La capacidad de juzgar determinante queda encerrada en categorías inflexibles que rigen su procedimiento y que en correspondencia sólo pueden hallar un mundo de leyes de la naturaleza. ${ }^{9}$

${ }^{7}$ Cf. I. Kant, Crítica de la razón pura. Trad. de Pedro Ribas. Madrid, Alfaguara, 1978, pp. 130-131, A97 [p. 161, vol. III]. De la tarea de la imaginación se encargará el siguiente punto. Además, este breve resumen del proceso realizado por la capacidad determinante puede confrontarse con: María Elvira Martínez Acuña, La articulación de los principios en el sistema crítico kantiano. Concordancia y finalidad. Navarra, EUNSA, 1996, pp. 15-27.

${ }^{8}$ En cuanto a esta distinción entre objetividad y verdad, confrontar Henry Allison, El idealismo trascendental de Kant: una interpretación y defensa. Trad. de Dulce María Granja. Barcelona, Anthropos, 1992, pp. 128-129.

9 "Gracias a dicho canon [el canon del entendimiento aparece en la Crítica de la razón pura] puede decirse que la Lógica trascendental tiene como labor propia el corregir y asegurar el Juicio [la capacidad de juzgar] en el uso del entendimiento puro mediante reglas determinantes" (M. E. Martínez Acuña, op. cit., p. 23). 
Esta formulación nominal de la distinción nos presenta un primer problema de comprensión, ya que no contempla el error en el juicio determinante. Esto se debe a que todo juicio de este tipo encuentra su verificación en la eficacia o ineficacia de su aplicación: corresponde a cierto control de la naturaleza basado en sus leyes. Toda excepción que salga de esta legalidad quedará excluida de poder ser formulada por un juicio de la capacidad de juzgar determinante. Su eficacia depende de su aplicación automática. La aplicación de esta capacidad resulta una imposición que no encuentra ninguna limitación, en cuanto al control de la naturaleza.

Otro problema que plantea la comprensión de esta distinción en su formulación nominal, radica en entender cómo puede la capacidad reflexionante tener un particular sin contar con la categoría que debe aplicarse. Parece una contradicción con lo dicho en la Crítica de la razón pura el que pueda haber un particular antes de la aplicación de su categoría.

Para resolver estos problemas proponemos entender la distinción en cuanto a la ejecución de la capacidad de juzgar y no en cuanto a los productos. Esto significa que entenderemos ambas ejecuciones, la de la capacidad determinante y la de la capacidad reflexionante, como aplicadas a un mismo juicio, en el caso del juicio determinante. La capacidad determinante aplica automáticamente las categorías. Ahora bien, si esta aplicación, como hemos visto, resulta automática, entonces es la aplicación de las categorías de un sujeto ciego que no puede ver más allá de su control de la naturaleza. De ahí que podamos, tal vez, considerar a la capacidad reflexionante como un vigilante de esta aplicación. La capacidad reflexionante atravesaría el juicio determinante, identificando el contexto de aplicación y verificándolo. ${ }^{10}$ Esto último brindaría validez al juicio determinante.

10 "La interpretación de la 'Crítica del Juicio' tendrá, pues, que empezar por entender que esta contraposición o distinción o división no es lo que su presentación nominal parece, pues, bajo la aparición de una división binaria en la que lo 'reflexionante' o la 'reflexión' serían uno de los términos, lo que en verdad se establece es el concepto de 'reflexión' como algo inherente a la capacidad de juicio en cuanto tal y en general; sólo porque hay en general 'reflexión', tiene sentido hablar de 'juicio' y 'capacidad de juicio'” (Felipe Martínez Marzoa, Desconocida raíz común. Estudio sobre la teoría kantiana de lo bello. Barcelona, Visor, 1987, p. 15). También puede verse esta interpretación en F. Martínez Marzoa, Releer a Kant. Barcelona, Anthropos, 1985, pp. 110119. Estamos de acuerdo con Martínez Marzoa en que el elemento que hace posible el juicio es la reflexión, pero no que esto haga de la distinción una concepción unívoca. Aquí pretendemos sostener que precisamente al ser inherente la reflexión al juicio puede distinguirse una división binaria en cuanto a las capacidades, en determinante y reflexionante, porque la propia reflexión tiene su posibilidad en una distinción también binaria. Esto se desarrollará en los puntos siguientes. $C f$. M. E. Martínez Acuña, op. cit., pp. 367-370. 
Podemos ejemplificar lo anterior recordando el cuento de Edgar Allan Poe La carta robada. Aunque los policías emplean los métodos (capacidad determinante) más avanzados para encontrar la carta, no pueden dar con ella, ya que sólo pueden ver lo que queda delimitado dentro de las reglas del mismo método. Estos métodos, como los principios, son ciegos ante los casos que no están contemplados bajo su previa regularidad. Sólo una forma de pensar que sale de estos límites (capacidad reflexionante) podrá encontrar la carta en el lugar más obvio y que no podía ser contemplado por el método. Una forma de pensar, que reflexiona más allá de los límites del método empleado, reconoce la situación en que se quiere emplear, y es la que puede distanciarse del método y así modificar su forma de proceder de acuerdo al caso particular.

Por consiguiente, en el juicio determinante deben aparecer las dos capacidades, como dos momentos de constitución del juicio. Ellas ofrecen al juicio objetividad, en tanto que capacidad determinante, y validez (verdad) en tanto que capacidad reflexionante. Por su parte, el juicio reflexionante sólo ofrece subjetividad, por ser únicamente resultado de la capacidad reflexionante. Esta capacidad no puede constituir un objeto para todos, sólo la pretensión de ser válido porque todos deberían pronunciarse de la misma manera si cumplen con su reflexión.

En síntesis, entendemos la distinción nominal como la presentación de dos modos de ejercicio de la capacidad de juzgar - la capacidad determinante y la capacidad reflexionante-, que se aplican en el caso del juicio determinante. Por lo tanto, el juicio determinante es resultado de las dos capacidades y no como parece decir literalmente la definición nominal, sólo de la capacidad determinante. A su vez el juicio reflexionante sólo es resultado de la capacidad reflexionante.

\section{Aplicación de la categoría para conformar el particular en un juicio que ofrece un concepto (capacidad determinante) y la posibilidad de hallar un particular antes de la categoría (capacidad reflexionante): esquematismo}

Esta sección pretende mostrar cómo la capacidad determinante aplica las categorías de forma automática, por lo que es necesaria la participación de la capacidad reflexionante en el juicio determinante. Como ya hemos dicho, la posibilidad de corrección depende de esta última capacidad. Así también explicaremos cómo es posible que lo particular se dé sin la aplicación inmediata de una categoría, tal como aparece definida la capacidad de juzgar reflexionante en su distinción de la capacidad de juzgar determinante. 
La aplicación de la categoría para conformar el particular de acuerdo al concepto, no es más que la realización de un juicio y se encuentra tematizada en la Crítica de la razón pura, en el capítulo del "Esquematismo de los conceptos puros del entendimiento". ${ }^{11}$ El tratamiento es abordado desde "una doctrina trascendental de la capacidad de juzgar, una doctrina que manifiesta la posibilidad de aplicar a los fenómenos en general los conceptos puros del entendimiento". ${ }^{12}$ Una cita presentada anteriormente decía que la capacidad de juzgar es la facultad de pensar lo particular como contenido en lo universal (bajo categorías). Por eso una doctrina trascendental que estudie a esta capacidad ofrecerá las condiciones de posibilidad para cumplir con dicha función.

Ya hemos visto que el análisis de la unidad única que constituye el conocimiento encuentra por abstracción que esta unidad está compuesta por dos elementos heterogéneos: en cuanto a las representaciones, son la intuición y la categoría; y en cuanto a las facultades que la producen, son la sensibilidad y el entendimiento. Es necesario encontrar una representación y una facultad que logre mediar entre una y otra. Este tercer elemento tiene que ser homogéneo a los otros dos para hacer posible, por un lado, la conformidad entre las representaciones y, por otro, la unión entre las facultades. La tercera representación no puede contener nada empírico, porque sería homogénea a la intuición empírica (diversidad de sensaciones) pero no a la categoría. Por lo tanto, ésta tiene que ser pura, conteniendo elementos que respondan a la categoría. Una representación con estas características -que corresponda a la intuición y a la categoría- sólo puede ser trascendental. Esto, su carácter trascendental, significa que sólo puede contar con las condiciones de posibilidad que pertenezcan a ambos (sensibilidad y entendimiento y sus correspondientes representaciones). Kant propone como tercera representación el esquema trascendental y como facultad que lo debe producir, además de mediar entre sensibilidad y entendimiento, la imaginación trascendental. ${ }^{13}$

${ }^{11}$ El Esquematismo constituye la primera parte de la "Doctrina trascendental de la capacidad de juzgar", que trata las condiciones sensibles que hacen posible el uso de las categorías; la segunda parte comprende los principios del entendimiento, y estos son juicios sintéticos que surgen a priori de las categorías y que sirven de base a todos los demás conocimientos a priori. Cf. I. Kant, Lógica, p. 116 [p. 541, vol. vI].

${ }^{12}$ I. Kant, Crítica de la razón pura, p. 182, A138, B177 [p. 187, vol. III]. Los fenómenos en general son entendidos como lo particular en terminología de la Crítica de la capacidad de juzgar, pues aunque lo hemos presentado como la intuición, también dijimos que esta intuición es una diversidad que tiene que ser sometida a la síntesis y así constituye un fenómeno y más tarde diremos que no es otra cosa que una palabra. "La tarea oficial asignada al 'Esquematismo' es explicar cómo las categorías, que tienen su origen en la naturaleza del entendimiento humano, pueden aplicarse a lo que es sensiblemente dado a la mente, es decir, a los fenómenos" (H. Allison, op. cit., p. 274).

${ }^{13}$ Cf. I. Kant, Crítica de la razón pura, p. 183, A138, B177 [pp.187-188, vol. III]. 


\section{El esquema trascendental}

Los esquemas son productos a priori de la "imaginación" y median entre categorías e intuiciones. La tarea de la imaginación es mediar entre la universalidad conceptual de la categoría y la particularidad empírica de la intuición empírica; también tiene que mediar entre la categoría (unidad) y la diversidad pura de la intuición pura. Esto lo hace aplicando la categoría a la condición más universal de la sensibilidad: la forma del tiempo. Por consiguiente, se llega a esta aplicación automáticamente, desde los instantes dados en la forma del tiempo (un ahora después de otro).

La intuición empírica es la que tiene como contenido una diversidad de sensaciones. Toda sensación es recibida en la sensibilidad por medio de dos sentidos: el sentido interno y el sentido externo. Ambos sentidos tienen su forma: el espacio para el sentido externo, donde se tiene la estructura de "uno al lado del otro", y el tiempo para el sentido interno, donde se tiene la estructura que consiste en "uno después de otro". Toda sensación, por provenir del exterior, debe pasar por el sentido externo para llegar al sentido interno. Las sensaciones internas en cambio sólo son recibidas por el sentido interno. En otras palabras, toda sensación ha de pasar por la forma del sentido interno: el tiempo. El tiempo es la última o más universal condición de la receptividad (sensibilidad), ya que hace posible la recepción de toda sensación.

El esquema encuentra en la forma del tiempo la condición que lo hace homogéneo a la sensación (intuición empírica) y a la diversidad formal de la intuición pura. Esta forma del tiempo -hemos dicho- es "uno después de otro": sucesión de instantes. Cada instante del tiempo constituye una unidad absoluta que es representada en el momento del tiempo que llamamos "ahora". Por eso la forma o estructura del tiempo es la sucesión de "ahoras". Una sensación está contenida en un "ahora" de esa estructura del tiempo, porque la sensación es el resultado de la afección que recibe la mente en un instante. Esta sensación no ofrece más que un grado de realidad y la intuición empírica está constituida por una diversidad de sensaciones. Por eso cada sensación obtiene en la estructura del tiempo un orden por el instante que ocupa en la intuición. El tiempo determina la representación desde el primer momento en que es afectada la mente, en tanto que el producto de esa afección se ordena bajo la estructura de la forma del tiempo como condición universal de toda recepción de la sensibilidad.

Hasta aquí el esquema logra su homogeneidad con respecto a la intuición empírica de la sensibilidad, en vista de las categorías del entendimiento (de la misma manera ocurre con la intuición pura). Su función es ofrecer una representación homogénea a la diversidad de la intuición en relación con la unidad 
de la categoría. Por eso, la categoría, como universal de la unidad, ofrece una regla que guiará tal homogeneidad.

El tiempo, como condición formal de la diversidad del sentido interno y, consiguientemente, de la conexión de todas las representaciones, contiene una diversidad a priori en la intuición pura. Ahora bien, una determinación trascendental del tiempo guarda homogeneidad con la categoría (que constituye la unidad de esa determinación) en la medida que es universal y en que está basada en la regla a priori. ${ }^{14}$

El entendimiento ofrece la dirección de la determinación del tiempo. Con la apercepción trascendental guía el entendimiento esta determinación, ya que esta determinación del tiempo sólo encuentra su regla como unidad de la diversidad (categoría) en la apercepción trascendental. La apercepción trascendental aplica la regla (categoría) al sintetizar aquella diversidad de instantes de la sucesión en la determinación del tiempo. La determinación del tiempo sólo ofrece una serie de "ahoras" ordenados bajo la forma de la sucesión (uno después de otro). Hasta que la apercepción trascendental sintetiza la diversidad de instantes, convierte dicha determinación, aquella sucesión de "ahoras", en pasado-presente-futuro y las sensaciones son conformadas en una representación conceptual. La regla surge en la aplicación de la apercepción trascendental, porque delimita la sucesión y la sintetiza en una única representación que constituye el juicio y así el concepto.

Esta regla (categoría), que es aplicada en la actividad de la apercepción trascendental al sintetizar los instantes de la sucesión, consiste en reconocerse como sujeto de la acción, es decir, que adquiere conciencia de sí mismo, como "yo" y como el mismo que está sintetizando cada uno de los instantes (ahoras). La apercepción trascendental puede formularse por medio del "yo pienso". Un yo pienso que al reconocerse pensando cada uno de los instantes permite delimitarlos bajo una identidad y así sintetizarlos. ${ }^{15}$

En conclusión, el esquema trascendental, para ser homogéneo con la intuición empírica (diversidad de sensaciones) y con la categoría (unidad universal), tiene que estar constituido por la determinación del tiempo y la apercepción trascendental. Pero el esquema trascendental, antes de cumplir con el proceso de conocimiento (que no es otro que el proceso de conceptualización que se logra en un juicio), no está tematizado y debe ser construido en este proceso. Puede ser construido - consiguiendo esta homogeneidad-,

${ }^{14}$ Idem.

${ }^{15} \mathrm{Cf}$. Miquel Bastons, Conocimiento y libertad. La teoría kantiana de la acción, Pamplona, EUNSA, 1989, pp. 215-217. 
gracias a que se cuenta con la categoría (regla) que ofrece la apercepción trascendental. Esta regla (categoría) exige cumplir con el proceso de conceptualización de todo particular que ha sido recibido: llevarlo a un juicio.

Es simplemente [el esquema trascendental] la síntesis pura, conforme a una regla de unidad conceptual -expresada por la categoría- y constituye un producto trascendental de la imaginación, producto que concierne a la determinación del sentido interno en general (de acuerdo con las condiciones de la forma de éste, el tiempo) en relación con todas las representaciones, en la medida en que éstas tienen que hallarse ligadas a priori en un concepto, conforme a la unidad de apercepción. ${ }^{16}$

\section{Imaginación trascendental e imaginación empírica}

El esquema trascendental es producido por la imaginación trascendental. Esta imaginación es la facultad que media entre la sensibilidad y el entendimiento. Ella se encarga de realizar la síntesis trascendental que constituye el esquema; es capaz de realizar dicha síntesis porque está determinada por la forma pura de la sensibilidad que es el tiempo, ${ }^{17}$ y está sujeta a la regla de la unidad (categoría) que ofrece la actividad del entendimiento con la apercepción trascendental. Esta categoría (regla) exige la síntesis de la diversidad que guía la determinación trascendental del tiempo. Mientras que la determinación trascendental del tiempo sólo fija la estructura ordenada de la diversidad; la categoría (regla) como concepto puro del entendimiento (universal) exige someter aquella estructura en la construcción del esquema. Puesto que la imaginación trascendental está sometida al entendimiento (actividad sintetizadora), su actividad debe realizar una síntesis -de acuerdo a la guía que le ofrece el entendimiento-, que produzca el esquema trascendental. ${ }^{18}$

Si la imaginación no está sometida a esta regla (categoría) del entendimiento, sólo produce una imagen y no llega a producir un esquema. "En sí mismo el esquema es siempre un simple producto de la imaginación. Pero, teniendo en cuenta que la síntesis de la imaginación no tiende a una intuición particular, sino a la unidad en la determinación de la sensibilidad, hay que distinguir entre el esquema y la imagen”. ${ }^{19}$ Esta imaginación es empírica y

${ }^{16}$ I. Kant, Crítica de la razón pura, p. 185, A142, B181 [p. 190].

${ }^{17}$ Aunque las formas son espacio y tiempo, este último ocupa un lugar privilegiado; ver inciso anterior.

${ }^{18} \mathrm{Cf}$. Nelly Festini Illich, La imaginación en la teoría kantiana del conocimiento. Lima, Universidad Nacional Mayor de San Marcos, 1990, p. 28.

${ }^{19}$ I. Kant, Crítica de la razón pura, p. 194, A140, B179 [p. 189]. 
reproduce la representación por medio de las leyes de asociación, sin la regla trascendental (síntesis de la apercepción trascendental) del entendimiento. Estas leyes de asociación son empíricas y se dan en la experiencia como representaciones que suelen sucederse o acompañarse. La base de estas leyes es contingente, puesto que su conexión depende de la experiencia. Por lo tanto, esta imagen es subjetiva, en tanto que depende de la situación contingente que la ha generado.

El esquema es producido por la imaginación trascendental (productiva) y la imagen por la imaginación empírica (reproductiva). La imaginación trascendental encuentra su fundamento en una regla trascendental que le ofrece el entendimiento, mientras que la imaginación empírica se funda en una ley empírica de asociación. Esta regla trascendental somete la síntesis de la imaginación a la actividad (espontaneidad) que dicta el entendimiento: la producción de un juicio y así de un concepto. En cambio, las leyes empíricas sólo limitan la asociación que realiza la imaginación de aquella diversidad que ofrece la determinación del tiempo bajo una sucesión de sensaciones, a la reproducción de la imagen dada por la sensibilidad bajo aquella estructura sucesiva. ${ }^{20}$

Debido a que las leyes de la asociación no regulan ninguna actividad sintetizadora, no permiten producir una representación, sino tan sólo delimitar aquello que se ha recibido del exterior. No obstante, esta asociación sólo es posible suponiendo una regla trascendental que permita guardar una coherencia entre lo que recibe y que asocia. Esto significa que para poder asociar debe suponerse una constante en aquello que se asocia, y ésta sólo puede encontrarse suponiendo la regla trascendental que permite dar unidad a la diversidad. La regla trascendental es la apercepción trascendental, que no constituye otra cosa que la espontaneidad o actividad sintetizadora del entendimiento.

Con lo dicho hasta ahora hemos encontrado la posibilidad de que lo particular se dé sin la aplicación inmediata de una categoría, sino sólo con la regla trascendental, tal como lo hallamos en la definición nominal de la capacidad de juzgar reflexionante. Pero todavía hace falta exponer cómo es que logramos ser conscientes de este particular, porque si lo fuéramos por medio de esta apercepción trascendental (regla trascendental) se aplicaría una categoría (regla) a este particular, como lo demostró la Crítica de la razón pura acerca de la capacidad de juzgar determinante.

La apercepción trascendental como espontaneidad o actividad sintetizadora del entendimiento aplica la categoría (regla), que no es más que la unidad formal de las formas de la sensibilidad -espacio y tiempo- a la diversidad de la intuición. Por su definición la capacidad de juzgar reflexionante no cuenta con una categoría (regla) previa al encuentro con el particular. Por lo tanto

${ }^{20}$ Cf. ibid., pp. 166-167, B152 [p. 149]. 
tampoco puede proceder aquí la apercepción trascendental para llevar a cabo esta aplicación. Como en este caso no contamos con la apercepción trascendental, tendremos que encontrar, por otra vía que no sea la determinada desde la categoría, la aplicación del juicio que permita tener el concepto del particular para poder ser conscientes de él.

\section{La reflexión como condición de posibilidad para hacer consciente (inteligible) la particularidad y poder distinguir entre juicio de per- cepción (conciencia de la particularidad) y juicio de experiencia}

Aquí queremos exponer la posibilidad de que a la "capacidad de juzgar reflexionante" le aparezca el particular, por medio de la distinción entre juicio de percepción y juicio de experiencia, que aparece en los Prolegómenos a toda metafísica futura que haya de poder presentarse como ciencia.

Hasta ahora hemos hablado del segundo, del juicio de experiencia, como el momento puesto en el juicio determinante por la capacidad de juzgar determinante. Ahora podremos atender al primero, el juicio de percepción, subrayando que Kant presenta la distinción para demostrar los elementos objetivos del juicio de experiencia, pero que en algún caso aparecería como juicio de experiencia desde su origen, y en otro sólo como un segundo momento, después de haber sido antes juicio de percepción. Si el juicio fuera sintético puro a priori, esto es, basado únicamente en sus condiciones de posibilidad (formas de la sensibilidad y categorías), sin nada empírico, entonces sería un juicio de experiencia como el de las matemáticas puras, que son apodícticos. Pero si en cambio fuera un juicio sintético a priori, estaría basado en sus condiciones de posibilidad, aunque no sería absolutamente independiente de una relación empírica con la realidad, como no lo son los juicios de la física. ${ }^{21}$ Este último, el juicio sintético a priori, supondría un primer momento del juicio como percepción y posteriormente llegaría a ser de experiencia. En este caso podría distinguirse lo subjetivo y lo objetivo como dos momentos del mismo juicio. El juicio de percepción sería un primer momento subjetivo y empírico, mientras que el segundo sería un segundo momento objetivo y trascendental (condiciones de posibilidad). En este último caso, la presencia de la "capacidad de juzgar reflexionante" aparecería desde el primer momento en que empieza a constituirse el juicio y permanecería después de su constitución para verificarlo. En el caso del juicio sintético $a$ priori puro aparecería la capacidad de juzgar reflexionante, después de rea-

${ }^{21}$ Cf. I. Kant, Prolegómenos a toda metafísica futura que haya de poder presentarse como ciencia, pp. 78-81 y 116-117. 
lizado el juicio, para verificarlo. No puede descartarse absolutamente la presencia de la capacidad de juzgar reflexionante, antes de comenzar este juicio sintético a priori puro, identificando que se trata de un tipo de juicio el que tiene que llevarse a cabo aquí y que es el que corresponde a las matemáticas puras. En los dos casos la capacidad de juzgar reflexionante aparece como una reflexión trascendental.

\section{La reflexión trascendental}

Kant distingue entre reflexión trascendental y reflexión lógica. La reflexión lógica sólo compara los conceptos entre sí, mientras que la reflexión trascendental compara la representación con la facultad cognoscitiva que la produce. La reflexión trascendental debe poder acompañar todo el proceso del conocimiento y así poder esperar las representaciones que constituyen una única representación en el conocimiento: el concepto. En la reflexión lógica sólo encontramos la relación necesaria entre los conceptos y, en cambio, en la reflexión trascendental encontramos el valor de cada representación de acuerdo con la facultad que lo produce en el proceso del conocimiento. ${ }^{22}$

\section{La apercepción originaria como posibilidad de la reflexión trascendental}

La reflexión es un acto del entendimiento que realiza la apercepción originaria. Mientras que la apercepción trascendental ofrece la regla trascendental que somete la diversidad a la actividad sintetizadora, la apercepción originaria sólo piensa aquella regla.

La apercepción es, como condición de posibilidad del conocimiento, una regla trascendental. Esta, la apercepción (en general), es la regla que tiene

${ }^{22}$ Cf. I. Kant, Crítica de la razón pura, p. 278, A262-263, B318-319 [pp. 286-287]. Hess Heinz-Jürgen define esta distinción así “'Reflexión' en el sentido general de la palabra (para Kant) puede caracterizarse simplemente como un acto del entendimiento y no de la sensibilidad. Ella comprende como aspectos principales: 1. La reflexión lógica abstrae en el entendimiento reflexivo (de todo contenido de la representación) y todo lo que corresponde a la facultad del conocimiento en cuanto al origen y a la estructura de la representación, y 2 . La reflexión trascendental revela el 'fundamento de posibilidad' de la diversidad de representaciones objetivas en una conciencia, al producir la 'comparación objetiva de las representaciones entre sí y a la vez las condiciones previas para la afirmación constitutiva del 'objeto posible'" (Hess HeinzJürgen, "Zu Kants Leibniz-Kritik in der 'Amphibolie der Reflexionsbegriffe', en Beiträge zur Kritik der reinen Vernunft, de Ingerborg Heidemann y Wolfgang Ritzel. Berlin, Walter de Gruyter, 1988, pp. 210-211). Traducción mía. 
que poder acompañar a toda representación y se formula como "yo pienso". A su vez, la apercepción, que no es otra cosa que una regla trascendental (condición de posibilidad del conocimiento), tiene dos usos, en tanto que apercepción trascendental y apercepción originaria.

Si la apercepción trascendental es el acto "yo pienso", entonces la apercepción originaria consiste en pensar en el yo que realiza ese acto. Ella, la apercepción originaria, señala la identidad del sujeto que realiza esa acción. La apercepción originaria representa al yo que piensa, con una identidad originaria que brinda la regla de aquella otra apercepción trascendental. En esto se halla la anterioridad lógica (necesidad trascendental como condición de posibilidad del conocimiento) de la apercepción originaria y no temporal en correspondencia a la apercepción trascendental. Por esta apercepción originaria puede pensarse a aquel yo, pues se basa en la espontaneidad del pensar que no está sujeta a las representaciones objetivas que produce la actividad sintetizadora. La espontaneidad del pensar pertenece al entendimiento puro que puede representar basándose únicamente en las reglas (categorías) de esta facultad, ya que la representación de la apercepción originaria es pura, sin contenido sensible, y por eso nunca rebasa el pensamiento. Esta falta de contenido sensible de la representación "yo pienso" de la apercepción originaria constituye una proposición analítica, que no expresa nada que esté contenido en el sujeto. Esta proposición sólo se extiende al yo que piensa, permitiendo representar su identidad, pero no conocerlo. ${ }^{23}$

La apercepción originaria es la unidad (como identidad del yo que piensa) que guía la síntesis. Aunque ambas, la apercepción originaria y la apercepción trascendental, constituyen la regla que ha de seguirse en el proceso de sintetización (juicio), pueden, sin embargo, distinguirse en que la apercepción originaria ofrece la unidad que guía a la sintetización (juicio) y por su parte, la apercepción trascendental exige el cumplimiento de la regla (categoría) en la intuición que somete a la imaginación trascendental a sintetizar la diversidad. La apercepción originaria es la última condición de posibilidad del proceso de conocimiento. Por eso la apercepción originaria no está condicionada por las categorías.

Como última condición de posibilidad del proceso de conocimiento, la apercepción originaria está incondicionada. No hay otra condición después de ella. Ni está condicionada a cumplir con el proceso de conocimiento. La apercepción originaria puede pensar, es decir, puede reflexionar. La apercepción originaria es la condición de posibilidad del conocimiento, pero al no estar condicionada a éste, es a su vez condición de posibilidad del pensar. Como última condición puede tomar distancia en relación con el proceso

${ }^{23}$ Cf. I. Kant, Crítica de la razón pura, p. 158, B138 [p. 140, vol. III]. 
de conocimiento y pensar. Este pensar que no sigue ningún interés específico, puede reflexionar sobre el propio proceso de conocimiento.

De acuerdo a lo anterior podemos afirmar que la apercepción originaria hace posible la reflexión. Si esta reflexión está presente en el proceso de conocimiento (en el proceso de juzgar), está obligada a seguir todo el proceso hasta que se obtenga el juicio determinante y así el concepto, dando cuenta del cumplimiento de las condiciones de este proceso para que se pueda atribuir objetividad.

Por eso la conciencia que se alcanza con esta reflexión es una conciencia de la validez objetiva del proceso de conocimiento, ya que advierte el cumplimiento de la regla trascendental que ha realizado la apercepción trascendental. No sólo adquiere conciencia de esta validez objetiva, sino también de la verdad del juicio, en tanto que puede afirmarse de él su carácter de juicio de experiencia. Esta conciencia reflexiva ofrece validez al juicio, revelando el proceso en que ha sido realizado, y constata que se ha cumplido con las condiciones que se exigen para que pueda tener validez. La conciencia reflexiva comprueba la correlación entre el juicio de experiencia y las leyes de la naturaleza. Porque la conciencia reflexiva no sólo comprueba la eficacia del juicio determinante, también puede tomar en cuenta las excepciones y así puede corregir su aplicación.

Si esta conciencia reflexiva no aparece una vez que se ha formulado el juicio para verificar que es un juicio de experiencia (su correspondencia con las leyes de la naturaleza), entonces sólo tendremos un juicio de percepción. Este juicio, el de percepción, sólo tiene una validez subjetiva, porque aunque hubiera cumplido con la regla del proceso de sintetización (la apercepción trascendental guiando la aplicación de las categorías), no tendría conciencia de su cumplimiento. La conciencia que tiene este juicio es inmediata al resultado de la aplicación de la regla. Por consiguiente, este resultado no es corroborado. El juicio de percepción sólo es posible por la imagen que produce la imaginación empírica, determinada por las leyes de asociación, pero sin tener presente la intención de la apercepción trascendental. La reflexión trascendental tiene que comprobar el cumplimiento de dicha intención en el juicio determinante por medio del correlato de lo que afirma este último con respecto a las leyes de la naturaleza.

Los conceptos del entendimiento son también pensados a priori con anterioridad a la experiencia y van destinados a ésta. Pero no contienen más que la unidad de la reflexión sobre los fenómenos en el sentido que éstos han de pertenecer necesariamente a una conciencia empírica. ${ }^{24}$

${ }^{24}$ Ibid., p. 308, A310, B366-367. Cf. M. E. Martínez Acuña, op. cit., pp. 53-54. 
En síntesis, la imaginación empírica realiza esta imagen por una mera asociación de las sensaciones dadas a la intuición empírica. Y esta asociación sólo es posible bajo la suposición de una regla trascendental que dé unidad a la diversidad. El influjo de la apercepción trascendental está presente en esta conciencia sin que lo advierta la conciencia reflexiva de la apercepción originaria. En este último caso, en donde no se ejerce la conciencia reflexiva, se obtiene un juicio de percepción y no puede alcanzarse la determinación de un concepto, sino tan sólo una palabra que refiere sin una determinación objetiva.

\section{La capacidad de juzgar reflexionante y su distinción de la capacidad de juzgar determinante}

Por lo que hemos expuesto hasta ahora, podemos afirmar que la capacidad de juzgar determinante construye juicios determinantes (juicios del conocimiento objetivo de la experiencia) con ayuda de la capacidad de juzgar reflexionante. Y que la capacidad de juzgar reflexionante construye por ella misma y sin ayuda alguna juicios reflexionantes.

La capacidad de juzgar determinante cuenta para llevar a cabo el juicio determinante con las intuiciones y con las categorías; pero éstas no son suficientes: también cuenta con la condición que permite subsumir unas en las otras. Esta condición es la propia capacidad de juzgar. Esta capacidad de juzgar en su ejercicio determinante -ya lo hemos dicho- requiere para poder subsumir la intuición en la categoría, de la imaginación empírica y de la imaginación trascendental; y así también de la apercepción trascendental y de la apercepción originaria. Esta última, la apercepción originaria, es la que hace posible la reflexión trascendental, que no es otra cosa que la participación en el juicio determinante de la capacidad de juzgar reflexionante. Según nuestra exposición, la capacidad de juzgar reflexionante atraviesa el proceso de construcción del juicio determinante. La capacidad de juzgar reflexionante se percata-como conciencia reflexiva- de la situación en la que se realiza el juicio sintético a priori (juicio que pertenece a los juicios determinantes): puede tener conciencia del contexto en que se aplicarán las categorías (por ejemplo las de relación y modalidad que dependen para su aplicación de la percepción de la existencia de los objetos). Para lograr esto, la capacidad de juzgar reflexionante tiene que poder tener conciencia del particular, que no es otra cosa que la relación entre propósito y contexto; dicho de otra manera, tener conciencia del particular antes de contar con la categoría que debe aplicar quien ejerce la capacidad de juzgar reflexionante. Además, esta capacidad de juzgar reflexionante verifica -como conciencia que puede reflexionar (vol- 
ver sobre los pasos andados)-, la correspondencia del juicio sintético a priori y el juicio sintético a priori puro (juicios que pertenecen a los determinantes) con la experiencia, esto es, con las leyes de la naturaleza. Además puede corregir la aplicación del juicio determinante porque puede comprender la excepción que no logra abarcar este juicio.

También hemos explicado, por medio del examen del juicio de percepción, la manera en que la capacidad de juzgar reflexionante se percata del contexto donde tendrán que aplicarse las categorías. En este último caso, cuando se obtiene un juicio de percepción, no puede alcanzarse la determinación de un concepto, sino tan sólo se tiene una palabra que refiere sin determinación objetiva. Ésta es la conciencia del particular que tiene en un primer momento la capacidad de juzgar reflexionante. Su conciencia reflexiva no advierte la aplicación de la regla trascendental, porque en este caso no precede la categoría que sintetiza la diversidad, sino que la misma conciencia reflexiva tiene que producirla. La palabra en la que reflexiona (piensa) la capacidad de juzgar reflexionante no determina al objeto, sino tan sólo piensa en la relación en que se encuentra el sujeto refiriéndose al objeto. Por lo tanto, la capacidad de juzgar reflexionante se dirige (reflexiona) al propio sujeto que realiza el juicio y no a la determinación del objeto. La conciencia reflexiva de la capacidad de juzgar reflexionante tiene que buscar la regla que da conformidad a la situación en la que se encuentra el sujeto en relación con el objeto.

Sólo la misma capacidad de juzgar reflexionante puede darse como ley un principio trascendental semejante, y no tomarlo de otra parte (porque de otro modo sería capacidad de juzgar determinante), ni prescribirlo a la naturaleza, porque la reflexión sobre las leyes de la naturaleza se rige según la naturaleza, y no ésta según las condiciones de acuerdo con las cuales nos esforzamos por adquirir un concepto de ella que es, en vista suya, del todo contingente. ${ }^{25}$

La capacidad de juzgar en todos los casos está urgida de una regla para referir aquel particular, porque su tarea es realizar juicios. Ella no es una facultad que produzca ninguna representación. Esta capacidad tan sólo está enlazando toda representación con su concepto general. Por lo tanto, la capacidad de juzgar, siempre que pretende objetividad, va unida a la reflexión trascen-

${ }^{25}$ I. Kant, Crítica de la facultad de juzgar, p. 91 [p. 88]. Cf. David Sobrevilla, "La estética de Kant", en D. Sobrevilla, Repensando la tradición occidental. Filosofía, historia y arte en el pensamiento alemán: exposición y crítica. Lima, Amaru, 1986, p. 29. Presenta estas distintas conciencias para cada respectivo juicio. 
dental. "Esta reflexión trascendental es un deber del que no puede librarse nadie que quiera formular juicios a priori sobre cosas". ${ }^{26}$ Si en cambio la capacidad de juzgar realiza un juicio reflexionante, subjetivo, pero con pretensiones de universalidad, entonces la reflexión trascendental tiene que buscar la regla que permita afirmar que todo sujeto en tal contexto hablaría de su situación de la misma manera. Esta universalidad no se sostiene en una objetividad, sino en un pretendido reconocimiento de una forma de igualdad para todos y que permita presuponer un acuerdo previo entre todos los sujetos.

En breve, la distinción entre capacidad de juzgar determinante y capacidad de juzgar reflexionante que pretende Kant en la "Introducción" de la Crítica de la capacidad de juzgar, encuentra el punto que la hace posible en la distinción entre apercepción trascendental y apercepción originaria que presenta en la "Deducción de los conceptos puros del entendimiento" de la Crítica de la razón pura. ${ }^{27}$ Esto se debe a que aquella distinción entre las ejecuciones de la capacidad de juzgar sólo encuentra cabida en la distinción anterior entre juicio de percepción y juicio de experiencia, que tiene su fundamento en la reflexión trascendental y que a su vez es posible por la distinción entre las apercepciones. Esta distinción es la que permite distinguir el ejercicio de esta capacidad determinante y la capacidad reflexionante. Pero queda por explicar cómo puede esta capacidad reflexionante generar su regla que no está dada con anterioridad.

\section{El principio que hace posible a la capacidad de juzgar reflexionante encontrar la regla conforme con lo particular}

La capacidad de juzgar procede de manera reflexionante para construir la regla que debe aplicar al particular (palabra) que tiene dado. Ella no cuenta con la apercepción trascendental como regla trascendental de aplicación (producción en la síntesis del juicio) del concepto. Sólo tiene la identidad de la apercepción originaria, la cual le permite reflexionar sobre sí misma, de tal manera que piensa aquel particular en relación con el sujeto que realiza el juicio en cada caso. La reflexión se encuentra a sí misma pensando en el particular, pero sin el acto sintetizador que le permite obtener un concepto. La capacidad de juzgar exige aquella regla para poder hacer inteligible el particular. ${ }^{28}$

${ }^{26}$ I. Kant, Crítica de la razón pura, p. 278, A263, B319 [p. 289].

${ }^{27}$ Cf. ibid., pp. 168-169, B155 [pp. 150-151, vol. III].

${ }^{28}$ Cf. I. Kant, Crítica de la facultad de juzgar, p. 199 [p. 95]. Así distingue estos principios H. W. Cassirer: "De cualquier modo es muy importante hacer una clara distinción entre el principio trascendental de la capacidad de juzgar y los principios 
La capacidad de juzgar en su ejercicio reflexionante construye un principio que cumple con la exigencia de aquella tarea que tenía impuesta. Dicho principio tiene a su vez la función de guiar a la capacidad de juzgar en su intento de comprensión de lo particular. Así como en el caso de la capacidad de juzgar determinante, la regla trascendental de la apercepción trascendental ofrece la unidad (categoría) que permite sintetizar a la diversidad, así también la capacidad de juzgar reflexionante ofrece una unidad de las leyes empíricas de la naturaleza, suponiendo un entendimiento suprafinito o arquetípico que posibilita la necesidad en el reino de la contingencia. Si este principio permite ordenar las leyes empíricas, por medio de una unidad sistemática, no podría ser simplemente lógico. Este principio tiene que ser trascendental porque la unidad sistemática de las distintas leyes empíricas en su sentido lógico (en su simple posibilidad de pensarla) implica en sí misma el que tal unidad no sólo pertenezca al pensamiento, sino también a la realidad. Si pudiera pensarse esta unidad sin relación con la realidad, entonces podríamos poner en duda la existencia de la racionalidad. ${ }^{29}$

Por consiguiente, la capacidad de juzgar reflexionante construye su principio a priori, que es lo que le permite encontrar lo particular. Pero este principio se ve forzado a extenderse para poder aplicar un concepto a dicho particular. La extensión de este principio se hace sin pretensiones de alcanzar un conocimiento, sino en los límites de la subjetividad de la reflexión. Estos límites de la subjetividad son tan sólo las pretensiones de universalidad, que buscan y exigen la posibilidad de llegar a un acuerdo en la comunicación del concepto. La extensión del principio se realiza de una manera "técnica", porque no está regulada por el esquema que permite unir a la intuición con la categoría produciendo un juicio determinante y así un concepto determinado o preciso. En este caso la extensión de este principio requiere de habilidad (prudencia que sólo puede ser dada por la reflexión), porque él no ofrece a priori el procedimiento más adecuado para realizar la aplicación. Tan sólo ha de extenderse en beneficio de nuestra propia capacidad de juzgar reflexionante. ${ }^{30}$

trascendentales del entendimiento. Tenemos que recordar que el entendimiento nos provee de reglas definitivas, mientras que la capacidad de juzgar no puede más que proveernos de un principio directriz, un principio que nos permite mirar por medio de conceptos empíricos" (H. W. Cassirer, A Commentary on Kant's Critique of Jugment. Nueva York, Barnes and Noble, 1988, p. 117). Traducción mía.

${ }^{29} \mathrm{Cf}$. Miguel López Antonio, La facultad de juzgar reflexionante en el sistema de la razón pura. Tesis. Madrid, Universidad Complutense de Madrid, 1990, pp. 55-66; Joachim Peter, Das tanszendentale Prinzip der Urteilskraft. Eine Untersuchung zur Funktion und Struktur der reflektierenden Urteilskraft bei Kant. Berlin, Walter Gruyter, 1992, p. 166.

${ }^{30} C f$. I. Kant, Crítica de la facultad de juzgar, p. 52 [p. 46]. 
En resumen, la capacidad de juzgar reflexionante pretende comprender el particular, sin tener una regla que le indique el procedimiento para comprenderlo, puesto que no contaba con la categoría que le indicara el procedimiento. La capacidad de juzgar reflexionante tiene que construir esta regla que le permita comprender el caso particular. Ella tan sólo posee un principio $a$ priori que le permite percatarse del caso particular al que quiere aplicar la categoría. Pero este principio también es subjetivo, puesto que lo tiene que suponer como posibilidad de igualdad entre los sujetos. Entonces debe extender de una manera técnica aquel principio a priori para cumplir con esta aplicación. Para esto tiene que suponer un entendimiento, en cuanto apercepción trascendental (aunque no sea nuestro entendimiento) que ofrezca la categoría de la actividad sintetizadora. Suponer esto no significa rebasar los límites subjetivos de la reflexión, puesto que no pretende el conocimiento ni la extensión de este entendimiento. Unicamente advierte la necesidad que tiene la capacidad de juzgar reflexionante de un orden para poder aplicar su categoría. Por ello se da una regla para sí y no para el particular.

Este principio extendido supone la adecuación de la capacidad de juzgar reflexionante al particular, de tal manera que en la diversidad de particulares pueda encontrarse afinidad para colocarse bajo conceptos. Ahí estos particulares se han entregado como palabras carentes de determinación y que no aseguran ningún conocimiento. Podrían considerarse estas palabras como un lenguaje compuesto de analogías y sin ninguna precisión. ${ }^{31}$ Estas palabras -que serán empíricas en tanto que refieren las sensaciones dadas en una imagen particular-, deberán referir clases de aquel particular y estas clases a su vez podrán englobarse bajo los géneros empíricos (leyes generales empíricas) hasta alcanzar un sistema empírico. En esta totalidad construida por la capacidad de juzgar reflexionante adquiere sentido la particularidad y puede comunicarse a los demás con pretensiones de universalidad.

Sólo cuando la capacidad reflexionante encuentre esta totalidad, podrá tener conciencia de este particular. Alcanzar su totalidad y su conciencia no son momentos distintos en el tiempo. El principio (totalidad) de la capacidad de juzgar es construido en relación con el particular y el particular es construido en relación con el principio (totalidad). Si el principio de la capacidad de juzgar reflexionante es la finalidad de la naturaleza, no debemos entender por ello otra cosa que la totalidad construida en relación con el particular: la

31 "Un conocimiento tal es el conocimiento por analogía, que no significa, como se entiende ordinariamente la palabra, una semejanza imperfecta entre dos cosas, sino una semejanza perfecta de dos relaciones entre cosas completamente desemejantes" (I. Kant, Prolegómenos a toda metafísica futura que haya de poder presentarse como ciencia, p. 267). 
capacidad de juzgar reflexionante sólo enlaza dos representaciones, totalidad y particularidad, y no puede ser objetivamente consciente de una o de otra antes de este enlace.

Por lo propuesto aquí el conocimiento objetivo sólo surge de la presencia de la capacidad de juzgar reflexionante en el enlace entre intuición y categoría. Este enlace, que constituye el juicio determinante, como ya hemos visto, es producto de una actividad sintetizadora dirigida por una regla trascendental que determina su realización. En el caso del juicio reflexionante, el enlace es producto de la capacidad de juzgar reflexionante y se lleva a cabo por medio de una actividad técnica: la reflexión. 